\title{
Vocal Function Following Frontolateral Laryngectomy
}

\author{
(D) Ziya Saltürk, (D) Onur Üstün, (D) Tolgar Lütfi Kumral, (D) Güler Berkiten, (D) Belgin Tutar, (D) Hüseyin Sarı, (D) Hasan Sami Bircan, \\ (1) Yavuz Uyar
}

University of Health Sciences Turkey, Prof. Dr. Cemil Taşçıoğlu City Hospital, Clinic of Otorhinolaryngology, Istanbul, Turkey

\section{Abstract}

Objective: The aim of this study is to evaluate smoothed cepstral peak point and laryngostrobosopic results in patients who underwent frontolateral laryngectomy (FLL).

Methods: Eight patients who underwent FLL with bilateral modified radical neck dissection, and who completed at least 12 months of followup, were included. All patients underwent laryngostroboscopic evaluation at study commencement; glottal closure was examined. Voice records were taken at fundamental frequency (Fo) and smoothed cepstral peak point were analysed. Voice handicap index-10 was requested to be completed. Ten healthy individual constituted control group. Results were compared.

Results: The smoothed cepstral peak points were between 1.81-2.42 in the FLL group and 4.6-6.06 in controls, a significant difference. The Fo ranged from 61 to $192.63 \mathrm{~Hz}$ in the FLL group and from 118.57 to $197.61 \mathrm{~Hz}$ in the control group, also a significant difference. Laryngostroboscopy revealed significant differences between-group in their closure. Seven patients had incomplete closure in FLL group and the entire control group had complete closure except one female patient who had posterior glottal gap $(p=0.01)$. Voice handicap index-10 results were between 31-40 in FLL group and 8-14 in control group and also revealed a significant difference.

Conclusion: FLL reduces smoothed cepstral peak point and affects the mucosal wave, reducing voice quality.

Keywords: Cepstral peak point, frontolateral laryngectomy, laryngostroboscopy, vocal quality, voice handicap index

\section{INTRODUCTION}

The aim of partial laryngectomy is to completely remove the diseased part of larynx with safe margins and to preserve function including phonation, swallowing, breathing, and stabilization (1). Modified frontolateral partial laryngectomy is a procedure which can be applied for selected early glottic carcinomas (T1 and T2) involving the anterior commissure (2). The following surgical reconstruction can be performed by bipedunculated sternohyoid muscle flap and perichondrium in order to provide bulky tissue replacing vocal folds or can be left to secondary healing $(2,3)$. Glottic closure and the vibratory margin is an essential part of voice production. One vocal fold and an anterior commissure are removed in frontolateral laryngectomy (FLL) and reconstruction methods can be applied to replace vocal quality.
"Cepstrum is described as a discrete Fourier transform of the logarithm power spectrum; i.e. it is a log power of a log power spectrum" $(4,5)$. The cepstral peak is the peak in the cepstrum with the highest amplitude. When a linear regression line that represents the average sound energy is drawn through the cepstrum, the distance from the cepstral peak to this linear regression line is termed the Cepstral Peak Prominence (CPP) (6). It is the measure of the degree of harmonic organization (4). Another measure is the smoothened CPP (SCPP) which represents the distance between the first harmonic peak and the point with equal quefrency on the regression line through the smoothed cepstrum. The logic behind this acoustic marker is that the more periodic voice signal displays a more welldefined harmonic configuration in the spectrum (i.e., the more harmonic the spectrum), and, as a result the cepstral peak will 
be more prominent. Since it was first introduced by Hillenbrand et al. (4) and Hillenbrand and Houde (5), it has been proven to be a reliable and valid tool for evaluation of voice quality (6). It was shown that CPP integrates measures of voice waveform and periodicity perturbations, be them either amplitude, frequency or noise (7).

The vocal aspects of FLL was studied in several studies $(1,3,8$ 10). Although voice changes were confirmed in all of the studies, there are some contradictory results $(3,10)$. On the other hand, none of the studies evaluated SCPP in this population, this study aimed to evaluate SCPP in FLL patients.

\section{METHODS}

International review board approval was obtained from Okmeydanı Training and Research Hospital Ethical Committee (48670771-514.10). Eight patients who underwent FLL between 2010 and 2017 and completed at least 12 months of follow up was included in the study. The vocal parameters were shown not to change significantly after 12 months (11). Eight healthy individuals including 1 female, matching the age and gender were constituted as the control group.

All of the patients had laryngostroboscopic evaluation at the beginning of the study. The glottal closure was evaluated.

All voices were recorded using an AKG D5 (Vienna, Austria) dynamic microphone and a Lexicon Alpha (Lexicon by Harman, USA) external sound card. The microphone was placed $5 \mathrm{~cm}$ from the lips, and after deep inspiration, patients were told to sound the vowel "a" in Turkish for as long as possible. Praat software (version 4.4.13; Boersma and Weenink, University of Amsterdam, Amsterdam, the Netherlands) was used to analyse recordings and the Z-tool (James Hillebrand and Western Michigan University) was employed for SCPP analyses. Laryngostroboscopic analysis was performed by Karl Storz Pulsar 2 (Tuttingen, Germany). 70-degree rigid telescope was used. Voice Handicap Index-10 (VHI-10) which was translated and validated by Kılıç et al. (12) was used.

\section{Statistical Analysis}

The results were compared using SPSS ver. 22 software for Windows (SPSS Inc., Chicago, IL, USA). As subject numbers were limited, we employed the Mann-Whitney U test for comparisons.

\section{RESULTS}

The mean age was $55.8 \pm 2.49$ years in the sCPP group and $57.6 \pm 4.22$ years in the control group; these did not differ significantly $(p=0.83)$. The $s C P P s$ were between $1.81-2.42$ in the FLL group and 4.6-6.06 in controls, a significant difference (Table 1) (Figure 1, 2). The fundamental frequency (Fo) ranged from 182.67 to $192.63 \mathrm{~Hz}$ in the FLL group and from 118.57 to $197.61 \mathrm{~Hz}$ in the control group, also a significant difference (Table 1). Laryngostroboscopy revealed significant differences between-group in their closure. Seven patients had incomplete closure in FLL group and all of the control group had complete closure except one female who had posterior glottal gap $(p=0.01)$. VHI-10 results were between 31-40 in FLL group and 8-14 in control group and also revealed a significant difference (Table 1).

\section{DISCUSSION}

T1b and T2 glottic tumors are the major indications of FLL. T2 tumors should not involve more than one-third of opposite vocal fold and may have a minimal extension to supra or subglottic regions (9). It has excellent oncological results which are comparable to radiotherapy (13). On the other hand, the vocal function is preserved and vocal folds can be replaced by bipedniculated suprahyoid muscle flap or perichondrial flap to achieve better function (9). Biacabe et al. (14) found that reconstruction resulted in better frequency and less granuloma and web formation. In contrary to this idea, Szmeja and Leszczyńska (15) concluded that scarred tissue resulted in better voice.

Our results showed that there is a significant deterioration in SCPP and VHI-10 results. As none of the patients had reconstruction, we were not able to compare reconstructed and non-reconstructed neoglottises.

So far, the evaluation of voice quality following FLL was studied in different aspects (1,3,8-10,14-17). Dedivitis et al. (10) studied the configuration of neoglottis effect on the auditory perception and found that the anterior commissure synechiea caused voice quality deterioration. On the other hand, Pfuetzenreiter et al. (3) found that anterior commissure synechia did not affect results

Table 1. Results of frontolateral laryngectomy and control group

\begin{tabular}{|l|l|l|l|}
\hline & FLL group & Control & $\mathbf{p}$ \\
\hline sCPP & $1.76(0.44)$ & $5.09(0.63)$ & 0.0009 \\
\hline Fo & $182.67(6.61)$ & $148.01(31.77)$ & 0.0103 \\
\hline Glottic closure & $\begin{array}{l}7 \text { incomplete } \\
1 \text { complete }\end{array}$ & 8 complete & 0.01 \\
\hline VHI-10 & $27.25(4.30)$ & $6(2.72)$ & 0.009 \\
\hline
\end{tabular}

FLL: Frontolateral laryngectomy, Fo: Fundamental frequency, VHI-10: Voice Handicap Index-10, sCPP: Smoothened cepstral peak prominence

Mann-Whitney $U$ test p:0.05 is significant 


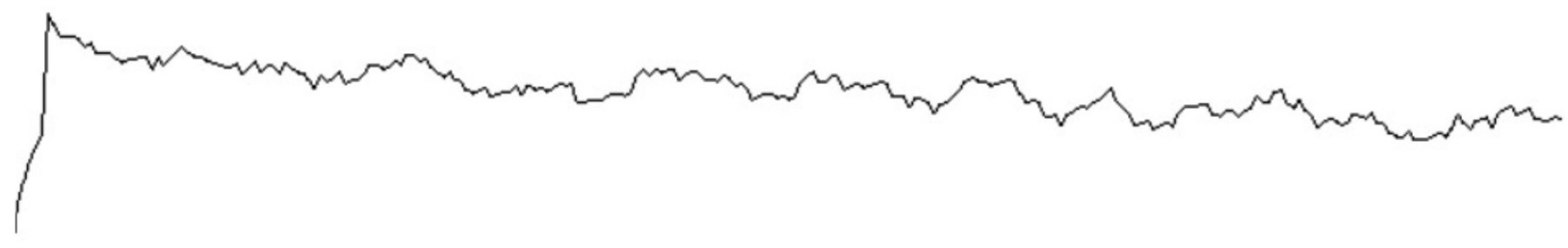

Figure 1. Smoothed cepstral analysis of frontolateral laryngectomy patients

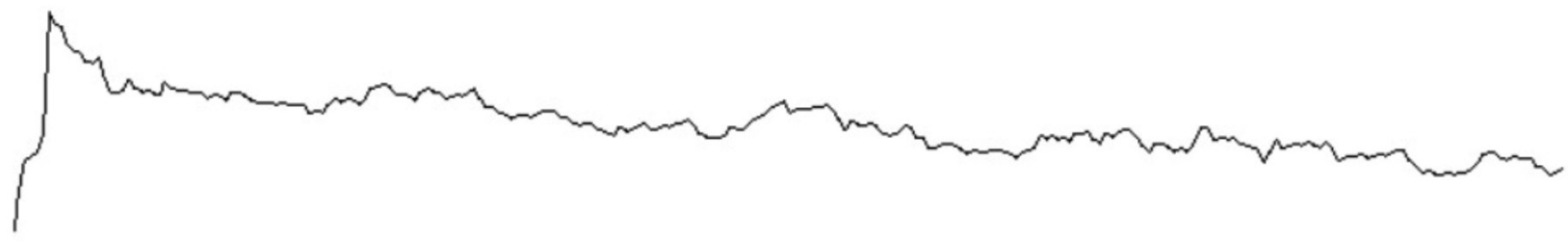

Figure 2. Smoothed cepstral analysis of healthy subject

of acoustic voice analysis. Regardless of synechiea, signals were chaotic and aperiodic. Because of the chaotic and irregular signals, we did not perform perturbation measurements in this study. Only Fo was evaluated and revealed a significant difference.

Cruz et al. (9) evaluated the stroboscopic data and found that 15 of the 21 neoglottis achieved complete closure. The vibration point was the glottis in 10 patients, supraglottis in 7 and mixed in 4 . They also pointed out that 5 reconstructed larynx had vibration but absent in the other 5. Dedivitis et al. (16) studied with videokymography and stated that 11 of 22 patients had a vibration at the glottis, 7 had it at the supraglottis and 4 had mixed vibration source. They also said that 4 of the 11 who had glottic vibration had no closed phase. Seven patients had an incomplete glottic closure which is one of the main factors in vocal deterioration.

The most important part of our study is the calculation of SCPP which is a better indicator of voice quality in patients who underwent FLL. It does not depend on frequency and aperiodic vibration is not a handicap for SCPP. It shows the overall voice quality better than any other measurement $(4-6,11)$. We also studied VHI-10 and found a significant difference between the groups.

The main disadvantage of our study was limited number of subjects. Moreover, none of the patients had reconstruction which prevented the comparison of it with the non-reconstructed counterpart. Because of irregular vibration pattern we did not use stroboscopic evaluation for the mucosal wave and we also did not analyze the source of vibration.

\section{CONCLUSION}

FLL reduces SCPP and causes deterioration in vocal quality. It affects both vibration and glottic closure. Further studies on larger cohorts are required to analyze not only the reconstructed but the non-reconstructed also.

\section{Ethics}

Ethics Committee Approval: Okmeydanı Training and Research Hospital Ethical Committee (48670771-514.10).

Informed Consent: Retrospective study. Approval was obtained from those who could be reached by phone.

Peer-review: Externally peer-reviewed.

\section{Authorship Contributions}

Surgical and Medical Practices: Y.U., Z.S., T.L.K, G.B., Concept: Z.S., O.Ü., Design: Z.S., O.Ü., Data Collection or Processing: O.Ü., B.T., Analysis or Interpretation: Z.S., T.L.K., H.S., Literature Search: H.S.B., O.Ü., Writing: Z.S., O.Ü., G.B.

Conflict of Interest: No conflict of interest was declared by the authors.

Financial Disclosure: The authors declared that this study received no financial support.

\section{REFERENCES}

1. Galant C, Lagier A, Vercasson C, Santini L, Dessi P, Giovanni A. A pilot study about speech changes after partial Tucker's laryngectomy: the reduction of regressive voicing assimilation. Eur Arch Otorhinolaryngol 2015;272:3843-9.

2. Dedivitis RA, Pfuetzenreiter EG, Guimaraes AV. Contact endoscopy of the larynx as an auxiliary method to the surgical margins in frontolateral laryngectomy. Acta Otorhinolaryngol Ital 2009;29:16-20. 
3. Pfuetzenreiter EG, Dedivitis RA, Queija DS, Bohn NP, Barros AP. The Relationship Between the Glottic Configuration After Frontolateral Laryngectomy and the Acoustic Voice Analysis. J Voice 2010;24:499-502.

4. Hillenbrand J, Cleveland RA, Ericsonn BR, et al. Acoustic Correlates of Breathy Vocal Quality. J Speech Hear Res 1994;37:769-78.

5. Hillenbrand J, Houde RA. Acoustic correlates of breathy vocal quality: dysphonic voices and continuous speech. J Speech Hear Res 1996;39:311-21.

6. Heman-Ackah YD, Sataloff RT, Laureyns G, Lurie D, Michael DD, Heuer R. Quantifying the cepstral peak prominence, a measure of dysphonia. J Voice 2014;28:783-8.

7. Fraile R, Godino-Llorente JI. Cepstral peak prominence: A comprehensive analysis. Biomed Signal Process Control 2014;14:42-54.

8. Kim CH, Lim YC, Kim K, Kim YH, Choi HS, Kim KM, et al. Vocal analysis after vertical partial laryngectomy. Yonsei Med J 2003;44:1034-9.

9. CruzWP, RapoportA, DedivitisRA, GuimarãesAV.Videolaryngostroboscopy following frontolateral laryngectomy with sternohyoid flap. Ann Otol Rhinol Laryngol 2004;113:124-7.

10. Dedivitis RA, Queija DS, Barros AP, Pfüetzenreiter EG Jr, Felippu A, Salvia $V F$, et al. The Impact of the Glottic Configuration After Frontolateral Laryngectomy on the Perceptual Voice Analysis: A Preliminary Study. J Voice 2008;22:760-4.
11. Makeieff M, Barbotte E, Giovanni A, Guerrier B. Acoustic and aerodynamic measurement of speech production after supracricoid partial laryngectomy. Laryngoscope 2005;115:546-51.

12. Kılıç MA, Okur E, Yıldııım İ, Öğüt F, Denizoğlu I, Kızılay A, et al. Ses Handikap Endeksi (Voice Handicap Index) Türkçe versiyonunun güvenilirliği ve geçerliliği. Kulak Burun Boğaz İhtisas Derg 2007;18:13947.

13. Giovanni A, Guelfucci B, Gras R, Yu P, Zanaret M. Partial frontolateral laryngectomy with epiglottic reconstruction for management of earlystage glottic carcinoma.Laryngoscope 2001;111:663-8.

14. Biacabe B, Crevier-Buchman L, Laccourreye O, Hans S, Brasnu D. Phonatory mechanisms after vertical partial laryngectomy with glottic reconstruction by false vocal fold flap. Ann Otol Rhinol Laryngol 2001;110:935-40.

15. Szmeja Z, Leszczyńska M. Voice function in patients after extended fronto-lateral laryngectomy. Eur Arch Otorhinolaryngol 1999;256:41822.

16. Dedivitis RA, Sertorio CB, Pfuetzenreiter EG Jr. Videokymographic analysis of patients after frontolateral laryngectomy with sternohyoid muscle flap reconstruction. Acta Otorhinolaryngol Ital. 2009;29:144-50.

17. Xu H, Kvit AA, Devine EE, Ying X, Dong P. Voice outcome of modified frontolateral partial laryngectomy in excised canine larynges and finite element model. Otolaryngol Head Neck Surg 2014;151:294-300. 